\title{
GOVERNANÇA COMPARTILHADA PARA REDES INTERORGANIZACIONAIS DE SEGURANÇA PÚBLICA: O CASE DA PARALIZAÇÃO DOS CAMINHONEIROS 2018
}

\author{
Djairlon Henrique Moura ${ }^{1}$ \\ Polícia Rodoviária Federal \\ djairlon.henrique@gmail.com \\ Patricia de Sá Freire ${ }^{2}$ \\ Universidade Federal de Santa Catarina \\ patriciadesafreire@gmail.com \\ Fernanda Kempner Kempner-Moreira ${ }^{3}$ \\ Universidade Federal de Santa Catarina \\ kempnereletrica@hotmail.com
}

\begin{abstract}
Resumo
O contexto da sociedade do conhecimento tem emergido a efetividade das Redes Interorganizacionais como estratégia para dar conta da crescente complexidade do ambiente organizacional. Para resultados efetivos essas Redes Interorganizacionais necessitam de uma governança que permita o alcance dos objetivos comuns, o que demanda o estabelecimento de mecanismos adequados Em um país cujo modal rodoviário é responsável por $80 \%$ da movimentação das riquezas, a paralização dos caminhoneiros em 2018 demandou articulação entre diversas organizações de segurança pública para atuar nesta situação complexa. Este artigo tem como objetivo analisar a influência dos mecanismos de governança das Redes Interorganizacionais para a melhoria da atuação da segurança pública em situações complexas. A análise do relatório produzido pelo Comitê de Crise da Polícia Rodoviária Federal (PRF) durante o movimento sinaliza a necessidade da formação de uma Rede Interorganizacional na segurança pública, a fim de uma atuação mais efetiva nas diversas situações complexas que envolvem este setor.
\end{abstract}

Palavras-chave: Redes Interorganizacionais. Governança compartilhada. Segurança Pública. Greve dos caminhoneiros.

\section{SHARED GOVERNANCE FOR INTER-ORGANIZATIONAL PUBLIC SAFETY NETWORKS: THE CASE OF TRUCK STOPPERS 2018}

\begin{abstract}
The context of the knowledge society has emerged the effectiveness of Interorganizational Networks as a strategy to cope with the increasing complexity of the organizational environment. For effective results, these Interorganizational Networks need a governance that allows the achievement of common objectives, which demands the establishment of adequate mechanisms In a country whose road modal is responsible for $80 \%$ of the movement of wealth, the paralysis of truck drivers in 2018 demanded articulation between various public security organizations to act in this complex situation. This article aims to analyze the influence of the governance mechanisms of the Interorganizational Networks to improve the performance of public security in complex situations. The analysis of the report produced by the Crisis Committee of the Federal Highway Police during the movement signals the need for the formation of an Interorganizational Network in public security, in order to act more effectively in the various complex situations involving this sector.
\end{abstract}

Keywords: Interorganizational Networks. Shared Governance. Public Safety. Trucking Strike.

\footnotetext{
${ }^{1}$ Chefe da Seção de Operações da Superintendência Regional no Rio Grande do Norte. Polícia Rodoviária Federal.

${ }^{2}$ Professora Doutora do Departamento de Pós-Graduação em Engenharia e Gestão do Conhecimento da Universidade Federal de Santa Catarina.

${ }^{3}$ Doutoranda do Programa de Pós-Graduação em Engenharia e Gestão do Conhecimento da Universidade Federal de Santa Catarina (EGC/UFSC)
}

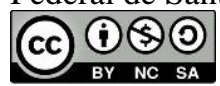

Esta obra está licenciada sob uma Licença Creative Commons (Attribution 4.0 International). P2P \& INOVAÇÃO, Rio de Janeiro, v. 6 n. 2, p.156-177, Mar./Ago. 2020. 


\section{INTRODUÇÃO}

A teoria de sistemas introduziu uma unidade complexa que não se resume à soma de suas partes. Um sistema complexo não é somente um determinado número de partes e as relações entre estas, mas um determinado número de diferenciações sistema/entorno que podem ser utilizadas operativamente e que, por sua vez, reconstroem de diferentes perspectivas o sistema global como unidade sistema/entorno (LUHMANN, 1997). Para Luhman (1997), os sistemas surgem como uma tentativa de reduzir a complexidade do entorno por meio da seleção entre as inúmeras possibilidades que este entorno proporciona (LIMA et al, 2018). Neste sentido, as organizações são construídas como consequência da redução da complexidade do entorno (LIMA; KEMPNER; TISCOSKI, 2010).

Os sistemas encontram-se expostos às perturbações provenientes do entorno que devem ser compensadas no seu interior. Nesta nova perspectiva paradigmática é possível entender as relações internas em termos de adequação ao entorno. A viabilidade dos sistemas se define pela capacidade de adaptação (RODRIGUEZ; ARLNOLD, 1991). Quanto maior a complexidade de um sistema, maior sua interação com o ambiente e sua capacidade de enfrentar a complexidade do próprio sistema (FREIRE et al, 2013). O objetivo é reduzir a complexidade com base em mecanismos seletivos com os quais se relacionam com seus ambientes (RODRIGUES; ARNOLD, 1991).

A compreensão da complexidade necessita de uma mudança profunda de nossas estruturas mentais, especialmente no que tange a entender que processos ambíguos são inseparáveis e interdependentes (MORIN, 2007). Como autonomia e dependência não são excludentes, a ideia de complexidade deve dar conta deste paradoxo. Quanto mais complexo um sistema, mais possibilidades ele terá para desenvolver sua autonomia. Igualmente, ele deverá estabelecer uma relação ainda maior de dependência com o seu entorno (BAUER, 1999).

Sistemas complexos são mais capazes de se adaptar às mudanças, de onde vem a expressão sistemas complexos adaptativos (MARIOTTI, 2002). O termo Sistemas Complexos Adaptativos (SCA) foi cunhado diante da percepção de que certos tipos de sistemas são capazes de responder, ativamente, aos acontecimentos ao seu redor (DUTRA; ERDMANN, 2006).

Em ambientes dinâmicos e altamente mutáveis, atuar em sistemas complexos adaptativos é uma estratégia para a manutenção da sustentabilidade e da competitividade organizacional. A compreensão das organizações parte da premissa de que elas são complexas, ambíguas e paradoxais, e que o grande desafio é aprender a lidar com essa complexidade (MORGAN, 1996). As organizações são muitas ao mesmo tempo, o que aumenta sua 
complexidade, e requer um senso de inter-relacionamento e integração entre os seus múltiplos contextos (ERDMANN; KEMPNER-MOREIRA, 2010).

O contexto da sociedade do conhecimento tem emergido a efetividade das Redes Interorganizacionais como estratégia para dar conta da complexidade crescente do ambiente organizacional. Atuar em redes tornou-se uma necessidade para que as organizações consigam inovar de maneira sustentável e, assim, enfrentar os desafios e a dinamicidade dos novos tempos. A atuação isolada não permite o alcance dos resultados desejados, sendo imperativo adentrar em aspectos de cocriação e coprodução, onde as relações têm um novo significado.

A paralisação nacional dos caminhoneiros em 2018, também chamada de Crise do Diesel, deflagrou uma crise nunca vista no país e impactou fortemente a segurança pública. Motivados pelos aumentos consecutivos no preço do diesel, o movimento paralisou e bloqueou estradas em 24 Estados do país, contingenciando recursos essenciais à população brasileira, o que provocou transtornos e prejuízos, além de uma sensação de instabilidade em toda a nação.

Em um país cujo modal rodoviário é responsável por $80 \%$ da movimentação das riquezas, esta paralisação impactou significativamente o abastecimento de recursos, alimentos, combustível, insumos hospitalares, entre outros, trazendo transtornos e prejuízos calculados em mais de 30 bilhões de reais gerados pela perda de produção econômica (CALEIRO, 2018).

A crise provocada pela paralisação nacional dos caminhoneiros apontou a necessidade de maior articulação para atuar em situações complexas, onde a solução e a gestão são dependentes da "relação" entre mais de duas organizações. "O governo brasileiro tem dificuldade de alinhar estratégia (o quê e por quê fazer) e planejamento (como fazer), prever acontecimentos e aplicar conhecimento relevante para resolver problemas complexos" (ANGELIS, 2015, p. 32), o que demanda atuação em rede.

Em dezembro de 2017, durante o evento RUASP/SUCEG, os gestores das academias do Sistema de Segurança Pública do Estado de Santa Catarina validaram a importância da formação em rede, e elencaram como principais desafios para esse trabalho: (i) ultrapassar as dificuldades de articulação entre as instituições; (ii) priorizar ações de prevenção e inteligência em detrimento da repressão. Angelis (2015) aponta outras barreiras no setor público, como a falta de interação estruturada entre as agências e a cultura dominante de que conhecimento relevante é poder e sua partilha deve ser cautelosa, entre outros.

Kim (2006) enfatiza a importância das parcerias, da coprodução, da formação de redes, programas de colaboração e projetos conjuntos. Parcerias interministeriais, interinstitucionais e público-privadas tornaram-se indispensáveis neste novo contexto de complexidade. As 
instituições públicas devem priorizar modelos de governança mais flexíveis, colaborativos e baseados em rede.

Especificamente para o setor público os benefícios gerados, proporcionados pelas Redes Interorganizacionais, são "aprendizado aprimorado, uso mais eficiente dos recursos e uma melhor capacidade de planejar e resolver problemas complexos" (HENTTONEN et al, 2016, p. 1). Na segurança pública as políticas são decorrentes de interação complexa e contínua entre atores internos e externos ao governo, fato que corrobora com a necessidade de formação de Redes Interorganizacionais para atender tamanha complexidade (TRINDADE, 2015).

Existe uma necessidade latente de formação de uma Rede Interorganizacional efetiva para a Segurança Pública, seja na esfera estadual ou federal, o que fica evidente pela criação do Sistema Único de Segurança Pública (Susp). Neste contexto, os estudos acadêmicos e científicos têm apontado diversas questões que demandam atenção dos centros de pesquisa e academias de polícia. Entre estas, o estudo que deu origem a este artigo buscou responder a seguinte pergunta: como os mecanismos de governança das Redes Interorganizacionais podem influenciar positivamente na atuação dos agentes de segurança pública em situações complexas?

Para responder a esta questão, definiu-se como objetivo deste artigo: analisar a influência dos mecanismos de governança das Redes Interorganizacionais para a melhoria da atuação da segurança pública em situações complexas. Esta análise baseia-se em um estudo realizado pela Polícia Rodoviária Federal (PRF), do caso do movimento da paralização dos caminhoneiros ocorrido em maio de 2018.

\section{FUNDAMENTAÇÃO TEÓRICA}

\subsection{Redes Interorganizacionais}

Uma rede interorganizacional é composta por diferentes organizações ligadas por laços multilaterais, conectadas por diferentes conexões, com o propósito de alcançar objetivos comuns (PROVAN; FISH; SYDOW, 2007). Quanto mais elos a rede desenvolve, mais complexas são suas relações, mais ela se desenvolve por meio dessas relações diversificadas e maiores são suas chances de sustentabilidade frente as contingências do entorno (CUNHA; PASSADOR; PASSADOR, 2011; FREIRE et al, 2013). Se a complexidade é o conjunto das múltiplas possibilidades (LUHMANN, 1997), a Rede Interorganizacional é a personificação de um sistema complexo. 
As Redes Interorganizacionais são formadas por objetivos diversos, sendo consenso que o objetivo inicial geralmente é a troca de informações e recursos (HARRIGAN, 1985; PODOLNY; PAGE, 1998; TRINDADE, 2015). Entretanto, essas redes podem evoluir para estágios mais complexos de relacionamento e estruturação. Kempner-Moreira, Freire e Souza (2019, no prelo) apontam cinco estágios de evolução das Redes Interorganizacionais: (1) Rede Interorganizacional de troca, onde o foco é o comprometimento na troca de recursos; (2) Rede Interorganizacional de desenvolvimento, instituindo-se parcerias técnicas e educativas; (3) Rede Interorganizacional de expansão, com o compartilhamento de informações, tecnologias e oportunidades; (4) Rede Interorganizacional de ação, onde busca-se desenvolver novos conhecimentos, produtos e atividades interorganizacionais; e a (5) Rede Interorganizacional de aprendizagem, cujo objetivo é a busca da aprendizagem em rede e, na própria rede. Esses estágios evoluem à medida que a rede fortalece seus canais de informação, transferência de conhecimento e aprendizagem coletiva, e desenvolve a interação sinérgica e a harmonização das capacidades de seus partícipes.

Não é imperativo que as redes evoluam. Entretanto, Redes Interorganizacionais são mais efetivas quando chegam ao nível de redes de aprendizagem. A rede de aprendizagem é considerada o estágio de desenvolvimento de uma Rede Interorganizacional em que as organizações interagem com objetivo explícito de aprenderem uns com os outros, por meio de novos tipos de cooperação com diferentes atores, transcendendo fronteiras e combinando diferentes tipos de conhecimento (KNIGHT, 2002; ALASOINI, 2018).

Neste estágio, os partícipes desfrutam do aprendizado proporcionado em e pela rede, o que amplifica o potencial de mudança transformadora, combinando inovações baseadas interações e intercâmbios entre os diversos atores (GOLDSTEIN; BUTLER, 2009, 2010; BUTLER; GOLDSTEIN, 2010; GOLDSTEIN, 2012). Ao avançar para a participação em redes de aprendizagem, a aprendizagem é elevada para o nível de transformação do indivíduo, das suas ações, das organizações e do impacto sobre a sociedade.

Estudos apontam para a necessidade da formação de redes em todos os setores, incluindo as organizações públicas. Trindade (2015) relata que, especialmente na segurança pública, a formação de Redes Interorganizacionais é uma estratégia inevitável para conduzir os problemas complexos do setor. Esse entendimento é corroborado pela formação do Sistema Único de Segurança Pública, que fomenta a formação de uma Rede Interorganizacional com as corporações que compõem a segurança pública no Brasil. 


\subsection{Rede Interorganizacional de Segurança Pública}

Redes Interorganizacionais são uma estratégia para ambientes de alta complexidade, como é o caso da segurança pública, pois esses ambientes exigem o envolvimento de múltiplas organizações e fornecem uma abordagem adaptável e flexível para questões complexas (TANG et al, 2017). É imperativo fomentar uma consciência compartilhada para estabelecer linhas de ação comuns no fornecimento de bens públicos (GARAU et al, 2017), pois uma resposta eficaz depende fortemente da colaboração (ANGELIS, 2015).

A nova gestão pública trouxe para o debate a importância da formação de Redes Interorganizacionais de coordenação e cooperação entre os atores envolvidos para a busca de soluções inovadoras aos problemas sociais e a criação de um futuro sustentável (KISSLER; HEIDEMANN, 2006). Esse debate reconhece que as Redes Interorganizacionais são uma estratégia inteligente para a sobrecarga de demandas da sociedade, que o setor público sozinho não consegue dar conta, necessitando não mais de governos, mas de governança (KIM, 2006; ANGELIS, 2015), como é o exemplo da segurança pública.

Para criar uma rede significativa e eficaz na administração pública, é necessário que o Estado tenha capacidade e disposição de coordenar várias atividades, sem perder a integridade estrutural do sistema de governo, o que transforma a Rede Interorganizacional no setor público um modo de regular a interdependência entre agências e outros atores sociais (KIM, 2006).

Entre os benefícios obtidos pela formação de Redes Interorganizacionais estão o aprendizado aprimorado, o uso mais eficiente dos recursos, a capacidade ampliada de planejar e resolver problemas complexos, o que requer trabalho efetivo de grande escala e de longa duração. Entre os desafios a serem superados estão a incerteza da tarefa, a comunicação muitas vezes virtualizada, as diferenças nos objetivos dos partícipes da rede e a diversidade cultural e organizacional, o que aumenta a complexidade da rede. Entretanto, até mesmo os desafios podem se tornar fatores de sucesso para a colaboração complexa (HENTTONEN et al, 2016).

As políticas públicas na área de segurança são decorrentes de interação complexa e contínua entre atores internos e externos ao governo (TRINDADE, 2015). A Lei 13.675 de 11 de junho de 2018 criou a Política Nacional de Segurança Pública e Defesa Social (PNSPDS), e instituiu o Sistema Único de Segurança Pública (Susp), “com a finalidade de preservação da ordem pública e da incolumidade das pessoas e do patrimônio, por meio da atuação conjunta, coordenada, sistêmica e integrada dos órgãos de segurança pública e defesa social da União, dos Estados, do Distrito Federal e dos Municípios, em articulação com a sociedade" (BRASIL, 2018). 
Entende-se que o Susp é uma tentativa de formar uma Rede Interorganizacional com as instituições que compõem a segurança pública nacional: Polícia Federal, Polícia Rodoviária Federal, Força Nacional de Segurança Pública, Secretarias de Segurança Pública, Polícia Civil, Polícia Militar, Corpo de Bombeiros Militar, Agentes Penitenciários, e Guardas Municipais. Isso não impede que outras instituições sejam agregadas à rede, como universidades, conselhos comunitários, entre outros.

Essa rede pretende gerar resultados a médio e longo prazo, já que a integração das instituições que a compõe é algo a ser realizado de maneira cuidadosa e respeitosa à autonomia de cada um dos integrantes. E como uma política de longo prazo, é preciso cautela às trocas de governo, devendo-se tornar uma política de Estado, ou seja, deve ter suas estratégias elaboradas por especialistas na área, que conhecem o contexto e as demandas específicas. Importante não estar associada a uma gestão, mas a uma temática, como é o caso da segurança pública.

Além dos órgãos integrantes do Susp, outros órgãos não necessariamente vinculados diretamente aos órgãos de segurança pública podem participar. Uma das medidas previstas é a integração do sistema de informações, com previsão de implantação por todos os órgãos de segurança pública do país em até dois anos.

A complexidade das Redes Interorganizacionais requer uma governança que leve seus partícipes a aprender, pois somente assim os resultados serão mais efetivos para todos os stakeholders. Neste sentido, parcerias interinstitucionais e público-privadas são partes indispensáveis de uma governança efetiva, que deve ser flexível, participativa e baseada em redes (KIM, 2006). Esse modelo de governança deve ser pautado em uma combinação equilibrada de governança relacional, contratual e hierárquica, como condição para a efetividade da rede (WILLEM; GEMMEL, 2013).

A complexidade das Redes Interorganizacionais requer uma governança compartilhada que leve seus partícipes muito além da simples troca de recursos e participação na tomada de decisão, mas à compreensão do processo de decisão e, consequentemente, à aprendizagem para autogovernar sua organização alinhada à governança coletiva, pois somente assim, os resultados serão mais efetivos para seus stakeholders. As organizações vivenciam um ambiente altamente complexo em termos econômico, social e político, o que reflete na necessidade da adoção de uma governança efetiva (ANSELL; GEYER, 2017). 


\subsection{Governança Compartilhada}

Por ser considerada um elemento-chave para as Redes Interorganizacionais (PROVAN; KENIS, 2008; WEGNER; KOETZ, 2016; WEGNER et al, 2018) a governança permite um maior comprometimento dos partícipes da rede com os resultados esperados, observando-se as razões pelas quais a rede foi formada, projetando uma estrutura que promova o crescimento do capital social e relacional tão indispensável para a continuidade da rede (ALVAREZ et al, 2010; WILLEM; GEMMEL, 2013; BERNSTEIN, 2015; TANG et al, 2017; STOREY et al, 2018).

O conjunto de mecanismos que sustenta e apoia essa interação, levando a rede e suas organizações a alcançarem as metas e os objetivos organizacionais pode ser definida como governança da Rede Interorganizacional (ALVAREZ et al, 2010; WEGNER; KOETZ, 2016; STOREY et al, 2018). Essa governança pode ser entendida como um processo emergente de aprendizagem, oportunizando a cocriação de conhecimento e cooperação dentro da própria rede (DE NONI et al, 2013; MASIELLO et al, 2015).

É necessário que as redes constituam uma governança adequada para que não apenas se assegure o cumprimento de acordos e contratos, mas estabeleça uma estrutura capaz de assegurar as condições e vínculos que ampliem o capital social e fomentem as relações e interações entre as organizações participantes (BERNSTEIN, 2015).

O modelo de governança compartilhado apresenta-se como o mais adequado para interesses coletivos da rede. Neste modelo o poder é simétrico, normalmente colegiado, em que as decisões são coletivamente construídas. Constitui-se de conexões densas e descentralizadas, onde a confiança é o principal mecanismo a ser desenvolvido (PROVAN; KENIS, 2008).

Redes que estabelecem a governança compartilhada são altamente flexíveis e adaptáveis às necessidades e expectativas de seus stakeholders, já que estes estão diretamente envolvidos nas decisões (PROVAN; KENIS, 2008). Essa governança fornece diversos benefícios, criando sinergias organizacionais que promovem o funcionamento mais eficaz por meio do compartilhamento de metas e utilização dos pontos fortes coletivos (KIM, 2006).

A eficácia da rede está atrelada à adequação dos mecanismos de governança com a estrutura de governança, elementos relacionados e interdependentes que podem existir em várias combinações (WILLEM; GEMMEL, 2013; ABEBE et al, 2017) e envolve um conjunto amplo de mecanismos que vão de estruturas formais a relacionais (ROEHRICH; LEWIS,2010; DE REUVER; BOUWMAN, 2012; LIN et al, 2012; KANG; ZAHEER, 2018).

Redes Interorganizacionais utilizam-se, basicamente, de dois grupos de mecanismos: os formais e os informais (SCARBROUGH; AMAESHI, 2009; ALVAREZ et al, 2010; LIN et al, 
2012; GURCAYLILAR-YENIDOGAN; WINDSPERGER, 2015; MASIELLO et al, 2015; CHANDRA; VAN HILLEGERSBERG, 2018)), também denominados, respectivamente, de mecanismos estruturais e relacionais (SCARBROUGH; AMAESHI, 2009; LEW et al, 2013; BERNSTEIN, 2015; MARTINS et al, 2017; STOREY et al, 2018; WEGNER et al, 2018).

Mecanismos formais são caracterizados pela formalização das relações entre os participantes da rede, e geralmente assumem a forma de sistemas de monitoramento, controles e relatórios por meio dos quais as organizações estruturam explicitamente sua interação. Tem como principal objetivo reduzir os riscos envolvidos nas transações em rede, controlar o oportunismo, coordenar as expectativas e comportamentos das organizações da rede e evitar a dissolução de colaborações Interorganizacionais. Procuram deixar claras e explícitas as regras de colaboração, interação e participação na rede. O mecanismo mais utilizado por esta tipologia são os contratos (YAER BANIHASHEMMI; LIU, 2012; BRITO; MIGUEL, 2017; CHANDRA; VAN HILLEGERSBERG, 2018).

Já os mecanismos informais são caraterizados por relacionamentos, e não pela estruturação burocrática. Estabelecem procedimentos flexíveis baseados na confiança e cooperação dos partícipes, o que pode ser mais adequado especialmente quando a formalização se apresenta onerosa e difícil (CHANDRA; VAN HILLEGERSBERG, 2018). São considerados pela literatura de relações interorganizacionais como os mecanismos mais utilizados, já que fomentam as relações e interações entre os partícipes (YASER BANIHASHEMI; LIU, 2012).

É praticamente consenso entre os autores que os dois tipos são complementares na governança de Redes Interorganizacionais (SCARBROUGH; AMAESHI, 2009; DE REUVER; BOUWMAN, 2012; LIN et al, 2012; MORAGUES-FAUS; ORTIZ-MIRANDA, 2012; YASER BANIHASHEMI; LIU, 2012; DE NONI et al, 2013; LEW et al, 2013; GURCAYLILAR-YENIDOGAN; WINDSPERGER, 2015; GARAU et al, 2017; MARTINS et al, 2017; TANG et al, 2017; CHANDRA; VAN HILLEGERSBERG, 2018).

Mecanismos de governança combinados, ou híbridos, podem melhorar o desempenho e criar maior valor para a rede ao buscar objetivos compartilhados (TANG et al, 2017). Mecanismos formais fornecem segurança e referência, enquanto os mecanismos informais mantêm a união e a interação na rede (COSTA; BIJLSMA-FRANKEMA, 2007; ALVAREZ et al, 2010; DE REUVER; BOUWMAN, 2012; LEE et al, 2012; GURCAYLILARYENIDOGAN; WINDSPERGER, 2015).

Sem a governança dos mecanismos formais, aumenta-se o risco de não se perpetuar os mecanismos informais nos relacionamentos. A complementariedade entre os mecanismos 
formais e informais da rede promove a aprendizagem e reduz conflitos (HERNANDEZESPALLARDO et al, 2010; KOHTAMÄKI, 2010; ROEHRICH; LEWIS, 2010; DE NONI et $a l$, 2013), facilitam a criação, a integração e o compartilhamento do conhecimento, aumentando a capacidade de governança da rede (GURCAYLILAR-YENIDOGAN; WINDSPERGER, 2015), e promovem a criação de novos conhecimentos, gerando inovação (SCARBROUGH; AMAESHI, 2009; LEW et al, 2013; MASIELLO et al, 2015).

Além disso, os mecanismos não são estáticos e devem ser continuamente revistos e reavaliados, a fim de garantir sua efetividade em uma rede dinâmica que evolui por meio de suas interações (COSTA; BIJLSMA-FRANKEMA, 2007; SCARBROUGH; AMAESHI, 2009; ALVAREZ et al, 2010; ROEHRICH; LEWIS, 2010; MASIELLO et al, 2015; BRITO; MIGUEL, 2017; KANG; ZAHEER, 2018). Isso requer uma governança igualmente dinâmica e atenta às necessidades da rede para manter os mecanismos adequados ao contexto e interesses de seus stakeholders, característica de uma governança compartilhada.

\section{PROCEDIMENTOS METODOLÓGICOS}

Este artigo é um estudo de caso do movimento dos caminhoneiros ocorrido em maio de 2018. Considerando que esse evento foi de solução complexa por parte da Segurança Pública Nacional, foi realizada uma pesquisa bibliográfica onde procurou-se evidenciar na literatura a importância das Redes Interorganizacionais e da governança compartilhada como estratégias para a solução de problemas complexos.

O caso dos caminhoneiros é apresentado com base nos documentos publicados pela imprensa nacional e internacional, em forma de relato jornalístico. A análise documental baseou-se em relatório produzido pelo Comitê de Crise da Polícia Rodoviária Federal durante o movimento dos caminhoneiros, elaborado pelo Coordenador de Planejamento e Controle Operacional, um dos autores deste artigo. $\mathrm{O}$ acesso à essa documentação foi autorizado pelo Diretor de Operações da Polícia Rodoviária Federal em fevereiro de 2019. Também foram realizadas entrevistas com profissionais que atuaram durante o movimento para maior profundidade da análise.

Salienta-se que outros órgãos que participaram do gabinete de crise instaurado pelo Governo Federal não foram consultados, sendo este artigo baseado exclusivamente no Relatório sobre a Paralisação no Setor de Transporte de Cargas entre 21/05 e 31/05/2018, produzido pela Coordenação de Planejamento e Controle Operacional da Polícia Rodoviária Federal, e em entrevistas com policiais rodoviários federais que atuaram no gabinete de crise desta instituição. 
Mas, pelo protagonismo da PRF na ação de desmobilização da paralização nas rodovias brasileiras e, a gestão para a diminuição do impacto negativo do movimento nos serviços essenciais, públicos e privados, este documento pode ser considerado crítico à análise proposta.

\section{O MOVIMENTO DOS CAMINHONEIROS: CRONOLOGIA E FATOS}

O chamado movimento dos caminhoneiros foi motivado pela nova política de preços da Petrobrás. Na primeira quinzena de maio a Associação Brasileira de Caminhoneiros (Abcam) cobrou, por meio de ofício enviado ao Governo Federal, a adoção de medidas efetivas contra o aumento constante das refinarias e redução dos impostos sobre o óleo diesel, sinalizando uma paralisação nacional caso não houvesse retorno (ECONOMIA IG, 2018).

A reivindicação era pela zeragem da alíquota do PIS/Cofins e pela isenção da Contribuição de Intervenção no Domínio Econômico (Cide), impostos que representavam quase metade do valor na refinaria. A diminuição desses impostos aliviaria o custo dos fretes, já que o diesel representa cerca de $42 \%$ desse valor (ESTADÃO, 2018). Outra reivindicação era a fixação de uma tabela mínima para os valores de frete (BBC BRASIL, 2018)

No dia 19 de maio entrou em vigor o quinto reajuste diário consecutivo nos combustíveis, o que fomentou o entendimento de que o Governo não se mostrou receptivo às demandas da categoria (G1, 2018). Em 21 de maio de 2018 os motoristas iniciaram as interdições totais ou parciais das estradas em, ao menos, 14 Estados brasileiros, expandindo-se para 24 Estados em apenas 1 dia (BBC BRASIL, 2018). Essa foi considerada a maior mobilização realizada pelo WhatsApp até então $(\mathrm{G} 1,2018)$.

Além das vias fechadas, o que começou a afetar a mobilidade, apesar de carros e outros veículos serem liberados, os primeiros sinais da paralisação começaram a ser sentidos já no segundo dia de paralisação: montadoras de veículos reduzem a produção por não receber os insumos esperados (BBC BRASIL, 2018) e os aeroportos começam a contingenciar combustíveis. Apesar de o governo anunciar acordos para zerar os impostos sobre o diesel e a Petrobrás reduzir temporariamente o preço dos combustíveis nas refinarias, o movimento não deu sinais de recuo $(\mathrm{G} 1,2018)$.

No terceiro dia de paralisação (23 de maio), o preço do combustível dispara nos postos, filas se formam com medo do desabastecimento, linhas de ônibus são reduzidas em diversas capitais, Correios suspendem postagens, linhas de produção de alimentos são paralisadas, observa-se escassez de hortifrutigranjeiros $(\mathrm{G} 1,2018)$. O governo pede uma trégua para buscar soluções, instaura um gabinete de crise, mas a paralisação continua (BBC BRASIL, 2018). 
Ao longo do movimento outras categorias e reivindicações foram somadas às bandeiras defendidas, como motoristas de vans e ubers, motoboys e agricultores, ganhando força os discursos anticorrupção (BBC BRASIL, 2018). O governo chega a anunciar um acordo no dia 24, o que é rejeitado pelas duas entidades que representam motoristas autônomos. O ministro da Segurança Pública afirma que há indícios de locaute, quando há influência de empresas do setor na paralisação, o que é proibido por lei $(\mathrm{G} 1,2018)$. A PRF, presente nas estradas, continua negociando para a liberação de produtos essenciais à sociedade.

No dia 25 a publicação de um decreto de Garantia da Lei e da Ordem (GLO) dá poder de polícia para as Forças Armadas em todo o país até o dia 04 de junho. Pela primeira vez essa operação GLO teve abrangência nacional. O Ministério da Defesa anuncia que a ação dos militares seria no intuito de garantir a distribuição de combustíveis em pontos críticos, proteger as infraestruturas críticas e desobstruir as vias próximas a refinarias de petróleo e centros de distribuição de combustíveis. Na prática o decreto autorizava a desbloquear as rodovias federais e, eventualmente, estaduais e municipais quando solicitadas, permitindo que integrantes das Forças Armadas ou da PRF dirigissem veículos até a garagem dos donos ou ao destino da carga. Multas por obstrução de estradas também foram realizadas pela Suprema Corte (BBC BRASIL, 2018). Com isso, as forças armadas se fazem presente nas estradas.

No sábado (26) os meios de comunicação começam a divulgar que 11 aeroportos estavam sem combustível em todo o país e, que, com o uso das Forças Armadas, os pontos de bloqueio começavam a ser desfeitos $(\mathrm{G} 1,2018)$. Ainda pela divulgação nas mídias, durante o domingo, o acordo para finalização do movimento é fechado, e a categoria entende que diversos pontos reivindicados foram atendidos. Entretanto, os reflexos da greve são sentidos especialmente pela população, com falta de combustível em $90 \%$ dos postos em diversos Estados (BBC BRASIL, 2018). Em ao menos 11 Estados o movimento foi mantido, e a produção agrícola sentiu fortemente os efeitos da paralisação. O governo anunciou redução de $\mathrm{R} \$$ 0,46 no preço do diesel por 60 dias, com ajustes a cada 30 dias após esse período (G1, 2018).

Na segunda-feira (28) a Confederação Nacional dos Transportadores Autônomos (CNTA) e o Sindicato Interestadual dos Caminhoneiros Autônomos ainda não davam por encerrado o movimento (G1, 2018). Porém, um levantamento da Polícia Rodoviária Federal (PRF) apontou um aumento no número de bloqueios, mas em apenas 3 pontos o trânsito estava interrompido. O número de manifestantes também havia diminuído, o que demonstrava que o movimento começava a perder força (BBC BRASIL, 2018).

Na quarta-feira (30), após dez dias de paralisação, a atuação das Forças Armadas e da PRF eliminou praticamente todos os pontos de concentração do movimento. Entretanto, os 
efeitos da greve, principalmente de desabastecimento, seriam sentidos pela população por alguns dias ainda (BBC BRASIL, 2018).

Além da redução de R \$ 0,46 no preço do óleo diesel, conseguidos por meio da zeragem da Cide e da redução da alíquota do PIS/Cofins, o governo publicou uma tabela que regulamentou os preços mínimos para o frete e isentou a cobrança de eixo suspenso de caminhões vazios em pedágios de todo o território nacional. Houve também a garantia de que a Companhia Nacional de Abastecimento (Conab) contrate 30\% de seus fretes com caminhoneiros autônomos (ESTADÃO, 2018).

\section{ANÁLISE DOS MECANISMOS DE GOVERNANÇA NO MOVIMENTO DOS CAMINHONEIROS}

A partir da análise do Relatório sobre a Paralisação no Setor de Transporte de Cargas entre 21/05 e 31/05/2018, produzido pela Coordenação de Planejamento e Controle Operacional da Polícia Rodoviária Federal, observa-se que, no início do movimento, o atendimento às demandas foi realizado por algumas instituições de maneira isolada, sem uma coordenação integrada. A PRF foi o órgão mais demandado, já que uma de suas atribuições é garantir a segurança e a fluidez das rodovias federais.

A instituição já estava de prontidão cerca de uma semana antes do início da paralisação, ocorrido em 21 de maio de 2018, orientando suas regionais a estabelecerem gabinetes de crises locais e estratégias conjuntas com as secretarias de segurança estaduais. No dia 24 de maio a PRF instalou um gabinete de crise nacional, buscando a articulação com diferentes stakeholders para minimizar os prejuízos decorrentes da paralização.

Esse gabinete de crise foi incorporado ao gabinete de crise político-estratégico, criado em 25 de maio com objetivo de resolver a crise com a utilização de outras ferramentas que não fossem as forças de segurança, como edição de medidas provisórias, negociação com o setor em greve, avaliação de todo o contexto, entre outros. Participaram deste gabinete representantes de todos os ministérios, Estado Maior das Forças Armadas, Polícia Rodoviária Federal, Polícia Federal e Secretaria Nacional de Segurança Pública.

Também em 25 de maio o Centro Integrado de Comando e Controle (CICCN) da Secretaria Nacional de Segurança Pública (Senasp), estabeleceu um gabinete de crise em nível tático, sob a coordenação da Diretoria de Operações da Senasp. Participaram deste gabinete representantes institucionais das forças de segurança em nível federal, representantes das forças 
de segurança dos Estados e de empresas privadas vinculadas ao setor de transporte de combustível.

Em 26 de maio, com a decretação da Garantia da Lei e da Ordem (GLO) pelo Presidente da República, esse gabinete foi transferido para as instalações do Ministério da Defesa, localizado na Esplanada dos Ministérios. O primeiro plano adotado na crise foi elaborado com informações coletadas e estruturadas pela PRF, por ser o órgão que dispunha de maior quantidade de informações naquele momento.

Além da PRF, órgãos de segurança pública do Governo Federal, como a Força Nacional de Segurança Pública, Exército Brasileiro, Marinha, Aeronáutica e Polícia Federal, o Centro de Operações Coordenadas do Ministério da Defesa contou com a participação de representantes institucionais da Presidência da República, Casa Civil, Agência Nacional do Petróleo (ANP), Ministério dos Transportes, Ministério da Saúde, Advocacia-Geral da União (AGU), Senasp, Agência Nacional de Aviação Civil (ANAC), entre outras, totalizando 23 agências atuando como atores e stakeholders da rede que se formou.

Após a deflagração da GLO a operação passou a ser coordenada pelo Ministério da Defesa e, rapidamente, as agências se moldaram à nova ordem, por compreender que a crise estava se agravando a cada momento e uma integração coordenada faria com que as ações fossem mais exitosas.

O gabinete de crise foi adequado a conceitos doutrinários de comando e controle, onde a interferência nos Estados se daria apenas em caso de necessidade apontada pela própria Superintendência Regional da PRF ou identificada pelo gabinete de crise da PRF no Ministério da Defesa (GAB/MD/PRF). Para melhor compreensão do que estava acontecendo em determinadas regiões foram criados grupos de mensagens instantâneas com seus respectivos responsáveis, para alinhamento do conhecimento e compreensão das necessidades dos Estados.

A integração da PRF com as Forças Armadas e outras forças de segurança priorizou o transporte de cargas para prover insumos de serviços essenciais, como combustível e medicamentos, por meio da realização de comboios. Entretanto, esta estratégia não se sustentaria por muito tempo, optando-se pela abertura de corredores de logística nas rodovias no dia 29 de maio.

Inicialmente todos os pontos de concentração foram abordados e os motoristas foram convidados a seguir viagem com escolta da PRF e Forças Armadas. Após abordagem dos pontos de concentração a PRF, Forças Armadas e Forças de Segurança de alguns Estados realizaram patrulhamento para impedir que os manifestantes provocassem novas abordagens a veículos de carga que estavam em deslocamento. Nos pontos de concentração onde os ânimos 
estavam mais exaltados equipes das Forças Armadas e PRF foram mantidas no local. No dia 31 de maio praticamente todos os pontos de concentração estavam desmobilizados.

Além da integração entre os órgãos que compuseram o gabinete de crise, observou-se que, em alguns Estados, as Secretarias de segurança pública foram provocadas a somar forças para controlar a situação. Destaca-se o apoio dado pela Polícia Militar e Polícia Civil do Estado da Bahia que realizaram diversas abordagens a pontos de concentração juntamente com a PRF, chegando até mesmo a realizar prisões. Esse apoio fortaleceu muito a ação e comprova a eficiência de uma Rede Interorganizacional.

A paralisação nacional dos caminhoneiros, ocorrida em maio de 2018, apesar de ter acontecido antes da promulgação da Lei 13.675/2018, propiciou diversas análises e aprendizagens para a formação da rede de segurança pública. O relatório produzido pela PRF aponta diversas lições aprendidas para que, em eventos futuros, o gerenciamento de crises possa partir da experiência vivenciada neste evento.

O fluxo informacional é um dos elementos a ser mais bem estruturado. Assim como o movimento dos caminhoneiros foi organizado por canais de comunicação rápida como o WhatsApp, a segurança pública também pode e deve se utilizar dessas ferramentas para atualizar e consolidar os dados com maior velocidade e padronização, agilizando a tomada de decisões. Melhorias no sistema de tecnologia de informação e comunicação também devem ser priorizados, com soluções interligadas entre as agências de segurança pública, como prevê a Lei 13.675/2018, especialmente no que tange a inteligência policial.

Apesar das agências terem atuado de forma mais integrada e coordenada a partir da formação do Gabinete de Crise no Ministério da Defesa, a pouca experiência e capacitação em situações similares trouxe, inicialmente, algumas dificuldades, superadas ao longo da crise. Neste ponto, espera-se que o Susp seja o motivador para a institucionalização de uma integração necessária para que a segurança pública nacional una forças na busca de soluções e objetivos comuns, além de estimular a capacitação dirigida ao gerenciamento de crises.

Em termos dos mecanismos de uma governança compartilhada, pode-se afirmar que alguns foram utilizados, enquanto outros precisam ser fortemente desenvolvidos. Ficou evidente que os mecanismos informais foram mais demandados, até por ainda não existir uma Rede Interorganizacional institucionalizada e consolidada entre os órgãos de segurança pública do país. Entretanto, sabe-se que é a utilização de mecanismos combinados que produz a maior efetividade nos resultados.

Como mecanismos informais observou-se a Cooperação, com ações conjuntas da PRF, Forças Armadas e Polícia Militar de alguns Estados; a Confiança entre as instituições de 
segurança pública para atuar em conjunto; o Compromisso de buscar soluções para o impasse do movimento; o Contato Pessoal e Informal e as Alianças Laterais, por meio de estratégias conjuntas com as Secretarias de Segurança Pública Estaduais e outros órgãos; a Troca de Informações e os Grupos Colaborativos com o uso do WhatsApp; a Autorregulação das regionais da PRF para atender demandas específicas e do próprio Gabinete de Crise do MD; os Acordos Informais Verbais com os líderes do movimento nos pontos de interdição; as Rotinas Colaborativas estabelecidas no Gabinete de Crise; o Capital Relacional criado, existente e fortalecido entre as instituições de segurança pública federais, estaduais e municipais, ministérios, empresas privadas e outros stakeholders; a Flexibilidade para compreender, atender e atuar frente às demandas do movimento.

Entre os mecanismos formais identificados estão os Contratos, por meio do Decreto de Garantia da Lei e da Ordem (GLO); as Alianças Estratégicas formadas juntamente com o Gabinete de Crise do MD; os Acordos estabelecidos para atuação conjunta do Exército e da PRF; a Estrutura de Comando que se formou a partir do Gabinete de Crise; as Reuniões e equipes regulares, também evidenciadas pelo Gabinete de Crise; a Autoridade Centralizada, a partir do momento que o Ministério da Defesa assumiu a responsabilidade e instituiu o Gabinete de Crise.

\section{CONSIDERAÇÕES FINAIS}

Entre os desafios para o avanço da governança compartilhada no setor público está o estabelecimento de parcerias, ou seja, a formação de Redes Interorganizacionais para maior interação e efetividade das ações públicas (ANGELIS, 2015).

Pelos estudos realizados com base nos relatórios, na participação do pesquisador no Comitê de crise e nas seguidas entrevistas, a formação de uma Rede Interorganizacional deve incluir não somente as organizações diretamente atuantes como órgãos da segurança pública, mas os diversos stakeholders inter-relacionados, sejam estes públicos ou privados. Exemplo dessa necessidade durante o movimento dos caminhoneiros foi a dificuldade de localizar veículos que transportavam cargas sensíveis, mesmo as empresas transportadoras dispondo de sistemas de rastreamento.

A dificuldade de diálogo, de atendimento prestativo ao comando, da disponibilização de dados e informações prejudicou o andamento e agilidade da aplicação das soluções. Em determinadas situações a participação de integrantes da iniciativa privada pode ser útil na 
compreensão do contexto e criação do conhecimento na busca de soluções. Identificar e gerenciar as relações entre os atores e stakeholders da rede, são desafios a serem superados.

Destaca-se que a ausência de um plano de gestão de eventos críticos e protocolos predefinidos provocou o retardamento de algumas decisões e certa insegurança por parte dos integrantes da instituição. As forças armadas, assim como toda e qualquer instituição, tem seu ritmo próprio e sua doutrina definida em manuais. Nesse caso específico, por ser uma ação que fugia de sua rotina, foi necessário o Estado Maior redigir regras de engajamento para cada tipo de ação, garantindo-lhes a tranquilidade jurídica para o emprego das tropas.

Não haviam protocolos para disponibilização de dados e informações à PRF também com as empresas privadas que precisaram ser chamadas a trabalhar em prol das soluções comuns. Se a Rede Interorganizacional de segurança pública estivesse consolidada, a ação poderia ter sido imediata e o engajamento das instituições mais efetivo.

Ainda hoje há rumores de novas paralisações dos caminhoneiros nas estradas brasileiras, o que deveria ensejar a elaboração de um plano de gerenciamento de eventos críticos com protocolos e ações preestabelecidas para cada partícipe e stakeholder da Rede Interorganizacional da Segurança Pública, bem como a designação de servidores para coordenar as iniciativas, com atribuições previamente definidas. Esses servidores devem estar orientados sobre fluxo informacional e atribuições para atuar em uma convocação emergencial de crise e, principalmente capacitados ao diálogo e negociação entre organizações de setores e comandos diversos.

Sendo assim, este estudo sugere a institucionalização dessa Rede Interorganizacional, com a normatização de integração e comando situacional definidos, e com todos os atores da segurança pública constituídos e conscientes de sua participação para a efetividade das ações da rede.

\section{AGRADECIMENTOS}

Reconhecemos com gratidão o apoio à pesquisa recebido do Programa UNIEDU PósGraduação da Secretaria da Educação do Estado de Santa Catarina. 


\section{REFERENCIAS}

ABEBE, G. K.; CHALAK, A.; ABIAD, M. G. The effect of governance mechanisms on food safety in the supply chain: Evidence from the Lebanese dairy sector. Journal of the Science of Food and Agriculture, [s. l.], v. 97, n. 9, p. 2908-2918, Jul 2017.

ALASOINI, T. Learning networks as a vehicle for improving the generative capacity of working-life development programmes. Action Research, [s. l.], v. 16, n. 1, p. 105-122, 2018.

ALVAREZ, G.; PILBEAM, C.; WILDING, R. Nestlé Nespresso AAA sustainable quality program: An investigation into the governance dynamics in a multi-stakeholder supply chain network. Supply Chain Management, [s. l.], v. 15, n. 2, p. 165-182, 2010.

ANGELIS, C. T. A Emergência da Reforma do Estado Brasileiro: governança compartilhada e o modelo do novo serviço público. Planejamento e políticas públicas, Brasília, n. 45, p. $13-46,2015$.

ANSELL, C.; GEYER, R. 'Pragmatic complexity'a new foundation for moving beyond 'evidence-based policy making'? Policy Studies, [s. l.], v. 38, n. 2, p. 149-167, 2017.

ATASHZADEH-SHOORIDEH, F. et al. Factors predisposing to shared governance: a qualitative study. BMC Nursing, [s. l.], v. 18, n. 1, p. 9, March 122019.

BAUER, R. Gestão da mudança: caos e complexidade nas organizações. São Paulo: Atlas, 1999.

BBC BRASIL. Greve dos Caminhoneiros: a cronologia dos 10 dias que pararam o Brasil. a cronologia dos 10 dias que pararam o Brasil. 2018. Disponível em:

https://www.bbc.com/portuguese/brasil-44302137. Acesso em: 30 mar 2019.

BERNSTEIN, L. Beyond relational contracts: Social capital and network governance in procurement contracts. Journal of Legal Analysis, [s. l.], v. 7, n. 2, p. 561-621, 2015.

BRASIL. Lei n ${ }^{\circ} 13.675$, de 11 de Junho de 2018. Institui o Sistema Único de Segurança Pública (Susp) e cria a Política Nacional de Segurança Pública e Defesa Social (PNSPDS),

Diário Oficial da União: seção: 1, Brasília, DF, Edição: 111, p. 4-8, jun. 2018.

BRITO, R. P.; MIGUEL, P. L. S. Power, Governance, and Value in Collaboration: Differences between Buyer and Supplier Perspectives. Journal of Supply Chain Management, [s. l.], v. 53, n. 2, p. 61-87, 2017.

BUTLER, W.; GOLDSTEIN, B. E. The US Fire Learning Network: springing a rigidity trap through multiscalar collaborative networks. Ecology and Society, [s. l.], v. 15, n. 3, 2010.

CHANDRA, D. R.; VAN HILLEGERSBERG, J. Governance of inter-organizational systems: a longitudinal case study of Rotterdam's Port Community System. Ijispm-International Journal of Information Systems and Project Management, [s. l.], v. 6, n. 2, p. 47-68, 2018. 
COSTA, A. C.; BIJLSMA-FRANKEMA, K. Trust and control interrelations - New perspectives on the trust-control nexus. Group \& Organization Management, [s. l.], v. 32, n. 4, p. 392-406, Aug 2007.

CUNHA, J. A. C.; PASSADOR, J. L.; PASSADOR, C. S. Recomendações e apontamentos para categorizações em pesquisas sobre redes interorganizacionais. Cadernos EBAPE. br, [s. l.], v. 9, p. 505-529, 2011.

DE NONI, I.; GANZAROLI, A.; ORSI, L. The evolution of OSS governance: a dimensional comparative analysis. Scandinavian Journal of Management, [s. l.], v. 29, n. 3, p. 247-263, Sep 2013.

DE REUVER, M.; BOUWMAN, H. Governance mechanisms for mobile service innovation in value networks. Journal of Business Research, [s. l.], v. 65, n. 3, p. 347-354, 2012.

DUTRA, F. A. F.; ERDMANN, R. H. Uma nova abordagem para o estudo do planejamento e controle da produção (PCP): a ótica da Teoria da Complexidade. Revista GEPROS, [s. l.], n. 1, p. 195, 2006.

ERDMANN, R. H.; KEMPNER-MOREIRA, F. Gestão eficiente de organização de saúde: do diagnóstico à melhoria da gestão. ENCONTRO NACIONAL DE PÓS-GRADUAÇÃO E PESQUISA EM ADMINISTRAÇÃO, 34., 2010, Rio de Janeiro. Anais eletrônicos [...]. Rio de Janeiro: EnANPAD, 2010. Disponivel em:

http://www.anpad.org.br/diversos/down_zips/53/gol2383.pdf. Acesso em: 19 mar. 2019.

FEIOCK, R. C. Metropolitan governance and institutional collective action. Urban Affairs Review, [s. l.], v. 44, n. 3, p. 356-377, 2009.

FREIRE, P. S. et al. Vantagem da complexidade: desenvolvimento organizacional para o enfrentamento da hipercompetitividade. Revista Espacios, Caracas, v. 34, n. 6, p. 14, 2013.

G1. Cronologia: Greve dos Caminhoneiros. 2018. Disponível em:

https://g1.globo.com/economia/noticia/cronologia-greve-dos-caminhoneiros.ghtml. Acesso em: 25 mar 2019.

GARAU, C.; BALLETTO, G.; MUNDULA, L. A Critical Reflection on Smart Governance in Italy: Definition and Challenges for a Sustainable Urban Regeneration. In: BISELLO, A. et al (ed.). Smart and Sustainable Planning for Cities and Regions: Results of Sspcr 2015, Switzerland: Spring, 2017. p. 235-250. (Green Energy and Technology).

GOLDSTEIN, B. E. Collaborative resilience: Moving through crisis to opportunity. Cambridge, MA: MIT Press, 2012.

GOLDSTEIN, B. E.; BUTLER, W. H.. Expanding the scope and impact of collaborative planning: combining multi-stakeholder collaboration and communities of practice in a learning network. Journal of the American Planning Association, [s. l.], v. 76, n. 2, p. 238$249,2010$. 
GURCAYLILAR-YENIDOGAN, T.; WINDSPERGER, J. Complementarity between formal and relational governance mechanisms in inter-organizational networks: Combining resourcebased and relational governance perspectives. In: WINDSPERGER, J. et al. (ed.). Interfirm Networks: Franchising, Cooperatives and Strategic Alliances. Switzerland: Spring, 2015. p. 229-248.

HARRIGAN, K. R. Strategies for Joint Ventures. Lexington Books: Lexington, MA, 1985.

HENTTONEN, K.; LAHIKAINEN, K.; JAUHIAINEN, T. Governance Mechanisms in Multi-Party Non-Profit Collaboration. Public Organization Review, [s. l.], v. 16, n. 1, p. 1$16,2016$.

HERNANDEZ-ESPALLARDO, M.; RODRIGUEZ-OREJUELA, A.; SANCHEZ-PEREZ, M. Inter-organizational governance, learning and performance in supply chains. Supply Chain Management-an International Journal, [s. l.], v. 15, n. 2, p. 101-114, 2010. ISSN 1359-8546.

KANG, R.; ZAHEER, A. Determinants of alliance partner choice: Network distance, managerial incentives, and board monitoring. Strategic Management Journal, [s. l.], v. 39, n. 10, p. 2745-2769, 2018.

KEMPNER-MOREIRA, F.; FREIRE, P.S.; SOUZA, J.A. Os Cinco Estágios de Evolução das Redes Interorganizacionais. Revista Organizações em Contexto, São Paulo, 2019. No prelo.

KIM, J. Networks, network governance, and networked networks. International Review of Public Administration, [s. l.], v. 11, n. 1, p. 19-34, 2006.

KNIGHT, L. Network learning: exploring learning by interorganizational networks. Human Relations, London, England, v. 55, n. 4, p. 427-454, 2002.

KISSLER, L.; HEIDEMANN, F. G. Governança pública: novo modelo regulatório para as relações entre Estado, mercado e sociedade? Revista de Administração Pública, Rio de Janeiro, v. 40, n. 3, p. 479-499, 2006.

KOHTAMÄKI, M. Relationship governance and learning in partnerships. Learning Organization, [s. l.], v. 17, n. 1, p. 41-57, 2010.

LEE, I. W.; FEIOCK, R. C.; LEE, Y. Competitors and Cooperators: A Micro-Level Analysis of Regional Economic Development Collaboration Networks. Public Administration Review, [s. l.], v. 72, n. 2, p. 253-262, 2012.

LEW, Y. K.; SINKOVICS, R. R.; KUIVALAINEN, O. Upstream internationalization process: Roles of social capital in creating exploratory capability and market performance. International Business Review, [s. l.], v. 22, n. 6, p. 1101-1120, 2013.

LIMA, C. R. M. et al. A Competência comunicativa na administração discursiva de organizações. Informação@ Profissões, Londrina, v. 7, n. 1, p. 03-30, 2018.

LIMA, C. M.; KEMPNER-MOREIRA, F.; TISCOSKI, G. P. Discurso e aprendizagem em organizações complexas. In: ENCONTRO DA ASSOCIAÇÃO NACIONAL DE PÓSGRADUAÇÃO E PESQUISA EM ADMINSITRAÇÃO, 34., 2010, Rio de Janeiro. Anais [...]. Rio de Janeiro: ANPAD, 2010. 
LIN, H. M. et al. How to manage strategic alliances in OEM-based industrial clusters: Network embeddedness and formal governance mechanisms. Industrial Marketing Management, [s. l.], v. 41, n. 3, p. 449-459, 2012.

LUHMANN, N. Novos desenvolvimentos na teoria dos sistemas. In: NEVES, C. E. B.; SAMIOS, E. M. B. Niklas Luhmann: a nova teoria dos sistemas. Porto Alegre: UFRGS, 1997.

MARIOTTI, H. As paixões do ego: complexidade, política e solidariedade. 2 ed. São Paulo: Palas Athena, 2002.

MARTINS, G. S. et al. Supply chain relationships: Exploring the effects of both relational and structural embeddedness on operational performance. International Journal of Procurement Management, [California], v. 10, n. 5, p. 639-664, 2017.

MASIELLO, B.; IZZO, F.; CANORO, C. The structural, relational and cognitive configuration of innovation networks between SMEs and public research organisations. International Small Business Journal, [California], v. 33, n. 2, p. 169-193, Mar 2015.

MORAGUES-FAUS, A. M.; ORTIZ-MIRANDA, D. Governing cooperative quality schemes Lessons from olive oil initiatives in Valencia (Spain). Outlook on Agriculture, [California], v. 41, n. 1, p. 27-33, Mar 2012.

MORGAN, G.. Imagens da organização. São Paulo: Atlas, 1996.

MORIN, E. Introdução ao pensamento complexo. Porto Alegre: Sulina, 2007.

PODOLNY, J. M.; PAGE, K. L. Network Forms of Organization. Annual Review of Sociology, [s. l.], v. 24, p. 57-76, 1998.

PORTAL IG. Greve de caminhoneiros colocou Petrobras em xeque e expôs dependência rodoviária. Gabriel Guedes. Brasil Econômico, 19 dez. 2018. Disponível em:

https://economia.ig.com.br/2018-12-19/greve-dos-caminhoneiros-retrospectiva.html. Acesso em: 19 mar. 2019.

PROVAN, K. G.; KENIS, P. Modes of Network Governance: Structure, Management, and Effectiveness. Journal of Public Administration Research and Theory, [s. l.], v. 18, n. 2, p. 229-252, 2008.

PROVAN, K. G.; FISH, A.; SYDOW, J. Interorganizational networks at the network level: A review of the empirical literature on whole networks. Journal of Management, Índia, v. 33, n. 3, p. 479-516, 2007.

RODRIGUEZ, D.; ARNOLD, M. Sociedad y teoría de sistemas. Santiago: Universitária, 1991.

ROEHRICH, J. K.; LEWIS, M. A. Towards a model of governance in complex (productservice) inter-organizational systems. Construction Management and Economics, [s. l.], v. 28, n. 11, p. 1155-1164, 2010. 
SCARBROUGH, H.; AMAESHI, K. Knowledge Governance for Open Innovation: Evidence from an EU R\&D Collaboration. In: FOSS, N. J.; MICHAILOVA, S. (ed.). Knowledge Governance: Processes and Perspectives, 2009. p. 220-246.

STOREY, C. et al. Governing embedded partner networks: Certification and partner communities in the IT sector. International Journal of Operations and Production Management, [s. l], v. 38, n. 9, p. 1709-1734, 2018.

TANG, P. et al. Leveraging intergovernmental and cross-sectoral networks to manage nuclear power plant accidents: A case study from China. Journal of Cleaner Production, [s. $l]$, v. 162, p. 1551-1566, 2017.

TRINDADE, Arthur. Estado, governança e segurança pública no Brasil: Uma análise das secretarias estaduais de Segurança Pública. Dilemas-Revista de Estudos de Conflito e Controle Social, Rio de Janeiro, v. 8, n. 4, p. 607-632, 2015.

WEGNER, D.; FACCIN, K.; DOLCI, P. C. Opening the black box of small-firm networks: Governance mechanisms and their impact on social capital. International Journal of Entrepreneurship and Small Business, [s. l], v. 35, n. 4, p. 559-578, 2018.

WEGNER, D.; KOETZ, C. The influence of network governance mechanisms on the performance of small firms. International Journal of Entrepreneurship and Small Business, [s. l], v. 27, n. 4, p. 463-479, 2016.

WILLEM, A.; GEMMEL, P. Do governance choices matter in health care networks?: An exploratory configuration study of health care networks. BMC Health Services Research, [New York], v. 13, n. 1, 2013.

YASER BANIHASHEMI, S.; LIU, L. Lean governance: A paradigm shift in interorganizational relationships (IORs) governance. CONFERENCE OF THE

INTERNATIONAL GROUP FOR LEAN CONSTRUCTION, 20., 2012. San Diego. Anais [...]. San Diego, 2012. Disponível em:

https://iglcstorage.blob.core.windows.net/papers/attachment-82034cde-8a9f-47d4-b744-

0128b9c7d84c.pdf. Acesso em: 12 dez. 2019. 